\title{
Evaluation of the chemosensitivity of primary cultured malignant melanoma cells using the collagen gel droplet-embedded culture drug sensitivity test
}

\author{
MIKI TANIOKA $^{1,5}$, ATSUSHI UTANI ${ }^{1}$, ERI ARAKI ${ }^{1}$, HIROKO FUJII ${ }^{1}$, SATOSHI KORE-EDA ${ }^{1}$, \\ TAKAO TACHIBANA ${ }^{2}$, TOSHIKAZU TAKANO ${ }^{3}$, HISAYUKI KOBAYASHI ${ }^{3}$, \\ HARUHIKO NAKAJIMA $^{4}$ and YOSHIKI MIYACHI ${ }^{1}$
}

\begin{abstract}
${ }^{1}$ Department of Dermatology, Graduate School of Medicine, Kyoto University, Kyoto 606-8507; ${ }^{2}$ Department of Dermatology, Shiga University of Medical Science, Shiga 520-2192; ${ }^{3}$ Life Science Laboratory, Nitta Gelatin Inc., Osaka 581-0024;

${ }^{4}$ Infectious Disease Testing Department, Mitsubishi Kagaku Medience Corporation, Chiba, Japan
\end{abstract}

Received July 30, 2009; Accepted October 6, 2009

DOI: $10.3892 /$ etm_00000011

\begin{abstract}
Malignant melanoma usually shows resistance to a standard chemotherapy regimen. A useful in vitro method to evaluate individual chemosensitivity is required to select effective anti-cancer drugs. This study aimed to establish in vitro tumor response testing for malignant melanoma. We determined the chemosensitivity of primary cultured melanoma cells using the collagen gel droplet-embedded culture drug sensitivity test (CD-DST). Nineteen tests were carried out for 15 cases of malignant melanoma. Primary cultured melanoma cells in collagen gel droplets were exposed to anti-cancer drugs, including cisplatin, adriamycin, dacarbazine, nimustine and vincristine. After a 7-day incubation in a serum-free medium, living melanoma cells in a collagen droplet were detected by image analysis after staining with Neutral red reagent. In vitro drug exposure conditions were determined to reproduce the value of the plasma area under the time-drug concentration curve in vivo. The rate of evaluation of the primary culture of melanoma cells was $78.9 \%$ (15/19 tests). The chemosensitivity of cisplatin, adriamycin, dacarbazine, nimustine and vincristine was $15,62,0,0$ and 62\%, respectively. Dacarbazine was not suitable for CD-DST due to its prodrug characteristics. The CD-DST method was able to evaluate the chemosensitivity of malignant melanoma to anti-cancer drugs in vitro. This method can also be applied to estimate the efficacy of newly developed anti-cancer drugs in vitro.
\end{abstract}

Correspondence to: Dr Miki Tanioka, Department of Dermatology, Graduate School of Medicine, Kyoto University, 54 ShogoinKawahara-cho, Sakyo-ku, Kyoto 606-8507, Japan

E-mail: mtanioka@fukui-med.jrc.or.jp

Present address: ${ }^{5}$ Fukui Red Cross Hospital, 2-4-1 Tukimi, Fukui 918-8501, Japan

Key words: chemosensitivity, malignant melanoma, collagen gel droplet-embedded culture drug sensitivity test

\section{Introduction}

Chemotherapy for metastatic malignant melanoma (MM) is challenging. In Japan, DAV-Feron therapy, which includes systemic dacarbazine, nimustine, vincristine administration and local intracutaneous interferon $\beta$ injection, is the standard chemotherapy for metastatic MM. For MM with resistance to DAV-Feron therapy, DAC-Tam therapy (systemic cisplatin, nimustine, dacarbazine and oral tamoxifen) is one of the standard choices. However, it is very difficult for an effective anti-cancer drug to be selected for MM patients since each drug has a low response rate of 10 to $20 \%(1,2)$. Therefore, establishment of a useful and tailor-made chemosensitivity test is crucial. In addition, it is important that a test satisfies the following criteria: i) a high rate of evaluation of the primary culture; ii) can be applied to an early MM lesion with a small amount of tumor; iii) the applied drug concentrations can mimic physiological levels; iv) able to evaluate an anti-cancer effect without contaminating fibroblasts. Collagen gel dropletembedded culture drug sensitivity test (CD-DST) satisfies the above-mentioned characteristics. In this report, we tried to adapt the CD-DST for Japanese patients with MM.

\section{Materials and methods}

Patient background. Nineteen samples from $15 \mathrm{MM}$ patients were examined after obtaining written informed consent (Table I). The patients were managed as anonymous samples, and personal information, except for clinical manifestations, were regarded as confidential. The Medical Ethics Committee of Kyoto University approved this study, which was conducted in accordance with the principles of the Declaration of Helsinki. Pathological types included five cases of superficial spreading melanoma, seven cases of acral lentigenous melanoma, one case of lentigo maligna melanoma and two cases of nodular melanoma (Table I).

Preparation of primary melanoma cells for testing of the in vitro tumor response. Fresh surgical specimens $\left(\sim 64 \mathrm{~mm}^{3}\right)$ 
Table I. Summary of the clinical characteristics and the results of the CD-DST.

\begin{tabular}{|c|c|c|c|c|c|c|c|}
\hline \multirow[b]{2}{*}{ Case no. } & \multirow[b]{2}{*}{ Tested lesion } & \multirow[b]{2}{*}{ Type } & \multirow[b]{2}{*}{ Cisplatin } & \multirow[b]{2}{*}{ Adriamycin } & \multicolumn{3}{|c|}{ DAV-Feron therapy } \\
\hline & & & & & Dacarbazine & Nimustine & Vincristine \\
\hline $1-1$ & Primary & SSM & $54.9^{\mathrm{a}}$ & 21.8 & 86.9 & 84.7 & 14.9 \\
\hline $1-2$ & Skin metastasis & SSM & 96.3 & 53.9 & 100.0 & 100.0 & 60.7 \\
\hline $2-1$ & Primary & SSM & UPC & UPC & UPC & UPC & UPC \\
\hline $2-2$ & Skin metastasis & SSM & 95.2 & 100.0 & 95.7 & 95.9 & 95.1 \\
\hline 3 & Primary & SSM & UPC & UPC & UPC & UPC & UPC \\
\hline $4-1$ & Primary & SSM & 83.1 & 49.3 & 100.0 & 100.0 & 35.0 \\
\hline $4-2$ & Skin metastasis & SSM & 70.2 & 39.2 & ND & ND & 29.5 \\
\hline $4-3$ & Skin metastasis & SSM & 100.0 & 96.9 & 99.1 & 96.5 & 88.1 \\
\hline 5 & Primary & SSM & 77.4 & 42.7 & 88.7 & 84.2 & 88.7 \\
\hline 6 & Primary & ALM & 62.8 & ND & 96.6 & 100.0 & 46.2 \\
\hline 7 & Primary & ALM & ND & ND & 97.9 & ND & ND \\
\hline 8 & Primary & ALM & UPC & UPC & UPC & UPC & UPC \\
\hline 9 & Primary & ALM & 92.2 & 79.4 & 93.9 & 70.7 & 97.9 \\
\hline 10 & Primary & ALM & 74.5 & 29.7 & 100.0 & 100.0 & 29.7 \\
\hline 11 & Primary & ALM & ND & 53.4 & 96.4 & ND & 55.6 \\
\hline 12 & Skin metastasis & ALM & 84.9 & 62.0 & ND & ND & ND \\
\hline 13 & Primary & LMM & 93.8 & 75.9 & 90.8 & 100.0 & 36.8 \\
\hline 14 & Primary & NM & UPC & UPC & UPC & UPC & UPC \\
\hline 15 & Primary & NM & 87.2 & 98.8 & 100.0 & 82.3 & 73.5 \\
\hline
\end{tabular}

SSM, superficial spreading melanoma; ALM, acral lentiginous melanoma; LMM, lentigo maligna melanoma; NM, nodular melanoma; ND, not determined; UPC, unsuccessful primary culture. 'Growth inhibition rate in vitro. Bold print indicates drug was regarded as 'effective'.

were minced into a paste, suspended in Hank's balanced saline solution and treated with Dispersion Enzyme Cocktail EZ (Nitta Gelatin Inc., Japan) at $37^{\circ} \mathrm{C}$ for $2 \mathrm{~h}$. After digestion, each sample was centrifuged at $900 \mathrm{x}$ g for $3 \mathrm{~min}$, the supernatant was removed, and the dispersed tumor cells were collected through an $80-\mu \mathrm{m}$ nylon mesh. The recovered cells were cultured for 12-48 $\mathrm{h}$ in a collagen gel-coated flask (CG-flask; Nitta Gelatin Inc.). The collagen gel in the CG-flask was dissolved in the cell dispersion in the Enzyme Cocktail EZ, and only viable cells that adhered to the collagen gel were collected and used for the CD-DST.

Collagen gel droplet-embedded culture drug sensitivity test $(C D-D S T)$. CD-DST was performed according to the methods described previously (3). Briefly, the prepared tumor cell suspension was added to a neutralized collagen solution at a final concentration of $1 \times 10^{5}$ cells $/ \mathrm{ml}$. Three drops of the collagen-cell mixture (30 $\mu \mathrm{l} / \mathrm{drop})$ were placed in each well of a 6-well plate on ice and allowed to gel at $37^{\circ} \mathrm{C}$ in a $\mathrm{CO}_{2}$ incubator. The final density was $\sim 3 \times 10^{3}$ cells/collagen gel droplet. One hour later, each well was overlaid with $4 \mathrm{ml}$ PCM-2 medium (Serum-free Medium; Nitta Gelatin Inc.), each anti-cancer drug was added and incubation was carried out for the following 7 days. At the end of the incubation, the cancer cell colonies in the collagen gel droplets were stained by Neutral red solution for $2 \mathrm{~h}$. Each collagen droplet was fixed with $10 \%$ neutral formalin buffer, washed in water, air dried and quantified by image analysis. The growth rates in the control incubations were calculated as the total cell number on day $7 /$ total cell number on day 0 . The in vitro sensitivity was expressed as the percentage of the $\mathrm{T} / \mathrm{C}$ ratio, where $\mathrm{T}$ is the total cell number in the treated group and $\mathrm{C}$ is the total cell number of the control group. When the T/C ratio was $<50 \%$, the drug sensitivity was regarded as 'high sensitive', while $>50 \%$ and $<65 \%$ was considered as 'borderline', and $>65 \%$ as 'low sensitive'.

Determination of concentrations of anti-cancer drugs. A concentration of an anti-cancer drug was determined by the 24-h area under the concentration-time curve, when it is administered at an ordinary dose for Japanese patients with MM (4-6). The concentrations of cisplatin, adriamycin, dacrbazine, nimustine and vincristine were calculated as 0.2, 0.02, $0.5,0.025$ and $0.01 \mu \mathrm{g} / \mathrm{ml}$, respectively.

Sensitivities of dacarbazine and nimustine against the B16 cell line in vitro. B16 cells, a mouse melanoma cell line, were cultured in DMEM containing 10\% fetal bovine serum. B16 cells were collected by a standard trypsin/EDTA method and cultured in a collagen gel droplet and exposed to various concentrations of dacarbazine and nimustine.

Chemotherapy and response assessment. Four patients who completed at least one cycle of sensitivity-directed chemotherapy were considered evaluable for response. Tumor response was assessed by computed tomography and/or magnetic resonance imaging in 12-week intervals and evaluated according to response evaluation criteria in solid tumors (7). 
A

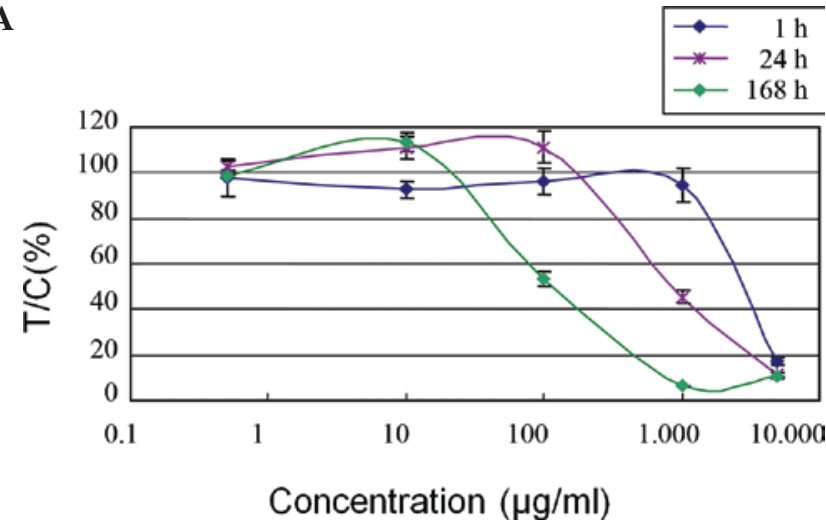

B

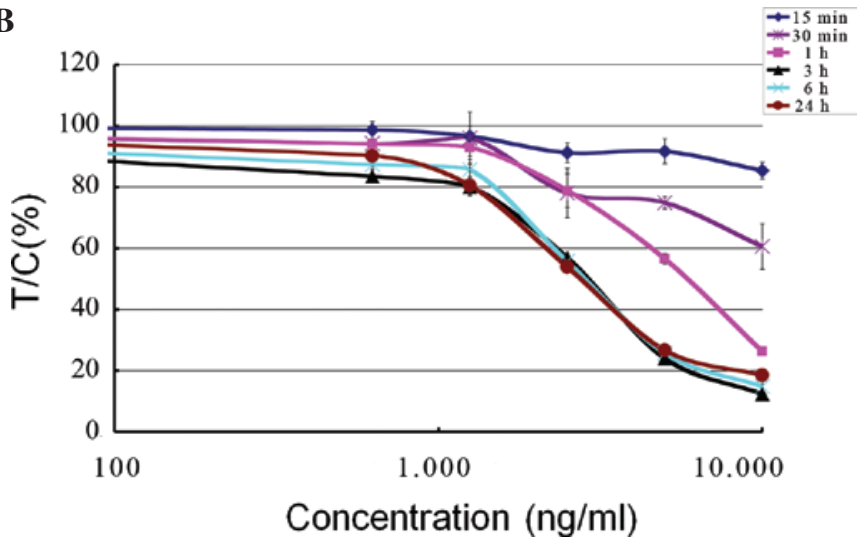

Figure 1. (A) Dose response curve of B16 cells against dacarbazine at various concentrations in the CD-DST. (B) Dose response curve of B16 cells against nimustine for various incubation times in the CD-DST.

\section{Results}

Evaluation rate of human primary melanoma cells. The overall evaluation rate in 19 tests was $78.9 \%$ (15/19 tests). The reasons for the unsuccessful four tests included one bacterial contamination, two cases having overly small specimens $\left(\sim 8 \mathrm{~mm}^{3}\right)$, and one case of non-viable cells due to resecting a sample after an effective chemotherapy (case no. 3). Multiple lung metastases of case no. 3 showed a complete response after administration of three courses of DAV-Feron therapy.

Chemosensitivities of human primary melanoma cells. When the 'high sensitive' and 'borderline' were regarded as 'effective', the in vitro chemosensitivity rate of cisplatin, adriamycin and vincristine were 15 (2/13), 62 (8/13) and 62\% (8/13), respectively. No cases showed sensitivity to dacarbazine and nimustine, which are standard drugs for treatment to malignant melanoma. Therefore, the chemosensitivity of B16 cells against nimustine and dacarbazine were evaluated using the CD-DST (Fig. 1A and B).

For nimustine, the B16 cells showed the same response curve at various incubation times. A 3-h incubation had the same antiproliferation effect compared with a $24-\mathrm{h}$ incubation. On the other hand, these cells showed no response to dacarbazine at physiological concentrations $(0.1-100 \mu \mathrm{g} / \mathrm{ml})$ in the CD-DST.

Correlation between the results of the CD-DST and clinical responses against anti-cancer drugs. We were able to evaluate the association of chemosensitivity with the clinical response in three cases. In case no. 1, the patient received chemotherapy of cisplatin and adriamycin for multiple lung metastases, according to the results of the CD-DST which showed high sensitivity to these agents (Table I, case no. 1-1). The lung metastatic tumors showed partial response; however, skin metastases appeared as recurrences, showing high resistance to cisplatin and adriamycin in the CD-DST (Table I, case no. 1-2). In case no. 2 , the CD-DST for the primary lesion was unsuccessful due to bacterial contamination. The patient was administered six courses of DAV-Feron therapy; however, skin metastases occurred, which showed high resistance to vincristine. In case no. 4 , the patient showed high sensitivity to adriamycin and vincristine in the CD-DST (Table I, case no. 4-1). After six courses of DAV-Feron therapy as adjuvant chemotherapy, skin and lung metastases appeared. The patient underwent chemotherapy with adriamycin and vincristine (AV therapy) according to the results of the CD-DST, and this treatment showed 'partial response' to the metastatic lung lesions and skin tumors. A chemosensitivity test of the recurred skin metastases after AV therapy showed high resistance to adriamycin and vincristine (Table I, case no. 4-3).

\section{Discussion}

The CD-DST method of treating MM cells in a threedimensional culture system in a collagen gel drop mimics tumors in the human body and enables MM cells to grow in vitro, resulting in a high success rate of primary culture of MM cells. In addition, serum-free culture for 7 days after drug exposure prevents the growth of fibroblasts. Moreover, contamination of fibroblasts is excluded by an image analysis, which detects the difference in cell shape and Neutral red staining between fibroblasts and MM cells. In many types of cancers including lung, breast, colon and gastric, the results of the CD-DST demonstrated good correlation with their clinical responses to anti-cancer drugs (8).

In this research, CD-DST exhibited a high success rate of primary culture of MM cells, which were obtained from small specimens of primary tumors. This suggests that the CD-DST method can evaluate the chemosensitivity of MM cells in a primary lesion and can also predict the effective anti-cancer drugs for future metastatic lesions in an internal organ when it is difficult to obtain a biopsy specimen.

The previously reported clinical response rate of MM cells against standard anti-cancer drugs is $10-20 \%$. Of these, dacarbazine is one of the most effective anti-cancer drugs for MM. In this study, dacarbazine showed no sensitivity in the CD-DST method. Thus, we speculate that dacarbazine is not suitable for CD-DST, since dacarbazine needs to be activated by the body's metabolism in order to produce diazomethane, a strong alkylating agent which imparts the major anti-cancer effect (9). Diazomethane is not suitable for in vitro evaluation of the efficacy of dacarbazine due to its explosive nature. Chemosensitivity data of B16 cells against dacarbazine also support its prodrug character. Nimstine is a typical drug, whose anti-tumor effect is dependent on the exposure concentration. Notably, the exposure time of $3 \mathrm{~h}$ may be necessary to obtain 
a sufficient anti-tumor effect according to our experimental data using B16 cell in vitro (Fig. 1B). This evidence allowed us to apply a higher concentration of nimustine on the MM cells when the time period was only $3 \mathrm{~h}$. Therefore, we plan to test a higher concentration of nimustine in our future CD-DST study.

In regards to the drug concentrations, the CD-DST can evaluate the chemosensitivity of tumor cells at a physiological concentration mimicking a normal blood concentration. In a large study of drug sensitivity-directed chemotherapy in metastatic melanoma using ATP-TCA assay, the concentration of cisplatin was $3.8 \mu \mathrm{g} / \mathrm{ml}$, compared with $0.2 \mu \mathrm{g} / \mathrm{ml}$ in the present study (10). We suggest that the collagen gel droplet reconstructs the minimum environment of the human body and realizes the genuine interaction between MM cells and drugs.

As for the correlation between clinical response and the results of the in vitro chemosensitivity tests, we evaluated only a small number of patients. However, a previous study reported that results of an in vitro chemosensitivity test predicted the clinical response and prolonged survival in patients with highly sensitive metastatic MM cells (10). To improve treatment outcome against MM, we believe that CD-DST might also be useful for predicting its clinical response. Further study of MM patients tested using CD-DST before and after chemotherapy is required to establish the clinical importance of CD-DST as well as a study of its value in other types of solid tumors (11).

In the future, we believe that the CD-DST method can be applied to evaluate new anti-cancer drugs against MM, since the concentration of anti-cancer drugs using the CD-DST method mimics physiological levels. In addition, a collagen drop reproduces the conditions in the human body. We are currently planning to assess new molecular target-based drugs against MM using the CD-DST.

\section{References}

1. Buzaid AC, Bedikian A and Houghton AN: Cutaneous Melanoma. 3rd edition. Quality Medical Publishing, St. Louis, pp405-418, 1998.

2. Balch CM, Reintgen DS, Kirkwood JN, et al: Cancer: Principles and Practice of Oncology. 5th edition. Lippincot-Raven Publishers, Philadelphia, pp1947-1994, 1997.

3. Kobayashi H, Tanisaka K, Doi O, et al: An in vitro chemosensitivity test for solid human tumors using collagen gel droplet embedded cultures. Int J Oncol 11: 449-455, 1997.

4. Mitsuhashi Y, Sugiyama Y, Ozawa S, et al: Prediction of ANCU plasma concentration-time profiles in humans by animal scale up. Cancer Chemother Pharmacol 27: 20-26, 1990.

5. Breithaupt H, Dammann A and Aigner K: Pharmacokinetics of dacarbazine (DITC) and its metabolite 5-aminoimidazole-4carboxamide (AIC) following different dose schedules. Cancer Chemother Pharmacol 9: 103-109, 1982.

6. Van den Berg HW, Desai ZR, Wilson R, et al: The pharmacokinetics of vincristine in man. Cancer Chemother Pharmacol 8: 215-219, 1982.

7. Therasse P, Arbuck SG, Eisenhauer EA, et al: New guidelines to evaluate the response to treatment in solid tumors. European Organization for Research and Treatment of Cancer, National Cancer Institute of the United States, National Cancer Institute of Canada. J Natl Cancer Inst 92: 205-216, 2000.

8. Tanigawa N, Kitaoka A, Yamakawa M, et al: In vitro chemosensitivity testing of human tumours by collagen gel droplet culture and image analysis. Anticancer Res 16: 1925-1930, 1996.

9. Bono VH: Studies on the mechanism of action of DTIC (NSC45388). Cancer Treat Rep 60: 141-148, 1976.

10. Ugurel S, Schadendorf D, Pfohler C, et al: In vitro drug sensitivity predicts response and survival after individualized sensitivitydirected chemotherapy in metastatic melanoma: a multicenter phase II trial of the Dermatologic Cooperative Oncology Group. Clin Cancer Res 12: 5454-5463, 2006.

11. Kobayashi H: Development of a new in vitro chemosensitivity test using collagen gel droplet-embedded culture and image analysis for clinical usefulness. Recent Results Cancer Res 161: 48-61, 2003. 